REVIEW ARTICLE

\title{
Nanoengineered targeting strategy for cancer immunotherapy
}

\author{
Wei-min Yin ${ }^{1}$, Yu-wei $\mathrm{Li}^{1}$, Yun-qing $\mathrm{Gu}^{1}$ and Min Luo ${ }^{1}$
}

\begin{abstract}
Cancer immunotherapy is rapidly changing the paradigm of cancer care and treatment by evoking host immunity to kill cancer cells. As clinical approval of checkpoint inhibitors (e.g., ipilimumab and pembrolizumab) has been accelerated by a dramatic improvement of long-term survival in a small subset of patients compared to conventional chemotherapy, growing interesting research has focused on immunotherapy. However, majority of patients have not benefited from checkpoint therapies that only partially remove the inhibition of T cell functions. Insufficient systemic T cell responses, low immunogenicity and the immunosuppressive environment of tumors, create great challenges on therapeutic efficiency. Nanotechnology can integrate multiple functions within controlled size and shape, and has been explored as a unique avenue for the development of cancer immunotherapy. In this review, we mainly address how nanoengineered vaccines can induce robust $T$ cell responses against tumors, as well as how nanomedicine can remodel the tumor immunosuppressive microenvironment to boost antitumor immune responses.
\end{abstract}

Keywords: cancer immunotherapy; nanomedicine; cancer vaccine; immune resistance; nanovaccine; immunosuppressive microenvironment

Acta Pharmacologica Sinica (2020) 41:902-910; https://doi.org/10.1038/s41401-020-0417-3

\section{INTRODUCTION}

Immunotherapy has been recognized as a novel and attractive treatment for cancer patients by boosting host immune responses to kill tumor cells [1]. In particular, approved immune checkpoint inhibitors (ICls) have shown considerable and long-lasting clinical benefit in several tumor types, targeting the suppression of T cells in cancer patients [2,3]. Clinical data has indicated that the overall response rate (ORR) to nivolumab in unresectable or metastatic melanoma patients was $31.7 \%$, compared to an ORR of $10.6 \%$ in those treated with chemotherapy [4]. However, a minority of patients (nearly $10 \%-30 \%$ response rates, depending on the type of cancer) respond to $\mathrm{ICls}[2,5]$. Insufficient systemic $\mathrm{T}$ cell responses in the majority of patients [6], failure of effector T cells to infiltrate into tumors or T cell exhaustion induced by the tumor microenvironment [7] limit antitumor immune responses. It is crucial to explore novel approaches to enhance tumor-specific $T$ cell responses and augment tumor-infiltrating lymphocytes (TILs) within the tumor microenvironment.

Nanomedicine has been extensively employed as a therapeutic in healthcare [8]. Nanoscale particles, such as liposomes, polymer nanoparticles (NPs) and micelles, show advantages for drugs delivery. Drugs encapsulated in polymer nanoparticles or chemical nanostructures can exhibit improved bioavailability and pharmacokinetic properties (e.g., Abranxane or Doxil) [9]. NPs also play an important role in the development of DNA- and RNA-based drugs. They have been used in the manufacture of Onpattro (patisiran, siRNA-containing lipid NPs for the treatment of transthyretinrelated hereditary amyloidosis) [10]. Proteins are another type of drug that can benefit from nanomedicine, such as the immunostimulatory agent interleukin-2, which can be nanoparticlized for cancer immunotherapy, with decreased systemic toxicity [11]. NP contrast agents can be used for MRI and ultrasound. Superparamagnetic iron oxide nanoparticles are employed as MRI agents that improve contrast and favorable biodistribution [12]. Some types of NPs can improve the mechanical properties and biocompatibility of biomaterials for medical implants and tissue engineering, such as nanomaterials exploited as dental fillers [13]. Furthermore, the properties of coencapsulation and the enhanced permeability and retention (EPR) effect improve the function of nanomedicine in multiple therapies [12].

In recent years, the application of nanomedicine in cancer immunotherapy has received much attention and holds tremendous promise. In particular, nanomaterial-based vaccines (nanovaccines) can target the lymph node system via subcutaneous injection, enhance antigen uptake and stimulate the tumorspecific $T$ cell response. Moreover, based on the EPR effect, nanomedicine can enhance drug accumulation in tumors via intravenous injection and remodel the immunosuppressive tumor microenvironment to boost the antitumor immune response. In this review, we discuss mainly how nanoengineering technologies provide innovative approaches for cancer immunotherapy, especially focusing on lymph node-targeting nanovaccines, as well as tumor microenvironment-targeting drug delivery.

\section{LYMPH NODE-TARGETING NANOVACCINES}

Unlike traditional vaccines, which boost the body's immune system to prevent infections, cancer vaccines boost the immune system to attack existing cancer cells [14]. Several anticancer vaccines have been processed in clinical trials, such as dendritic

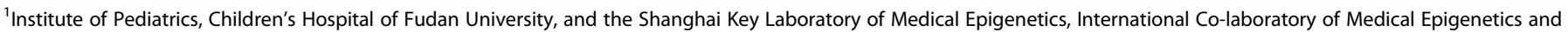
Metabolism, Ministry of Science and Technology, Institutes of Biomedical Sciences, Fudan University, Shanghai 200032, China

Correspondence: Min Luo (luo_min@fudan.edu.cn)

Received: 12 December 2019 Accepted: 12 April 2020

Published online: 12 May 2020 


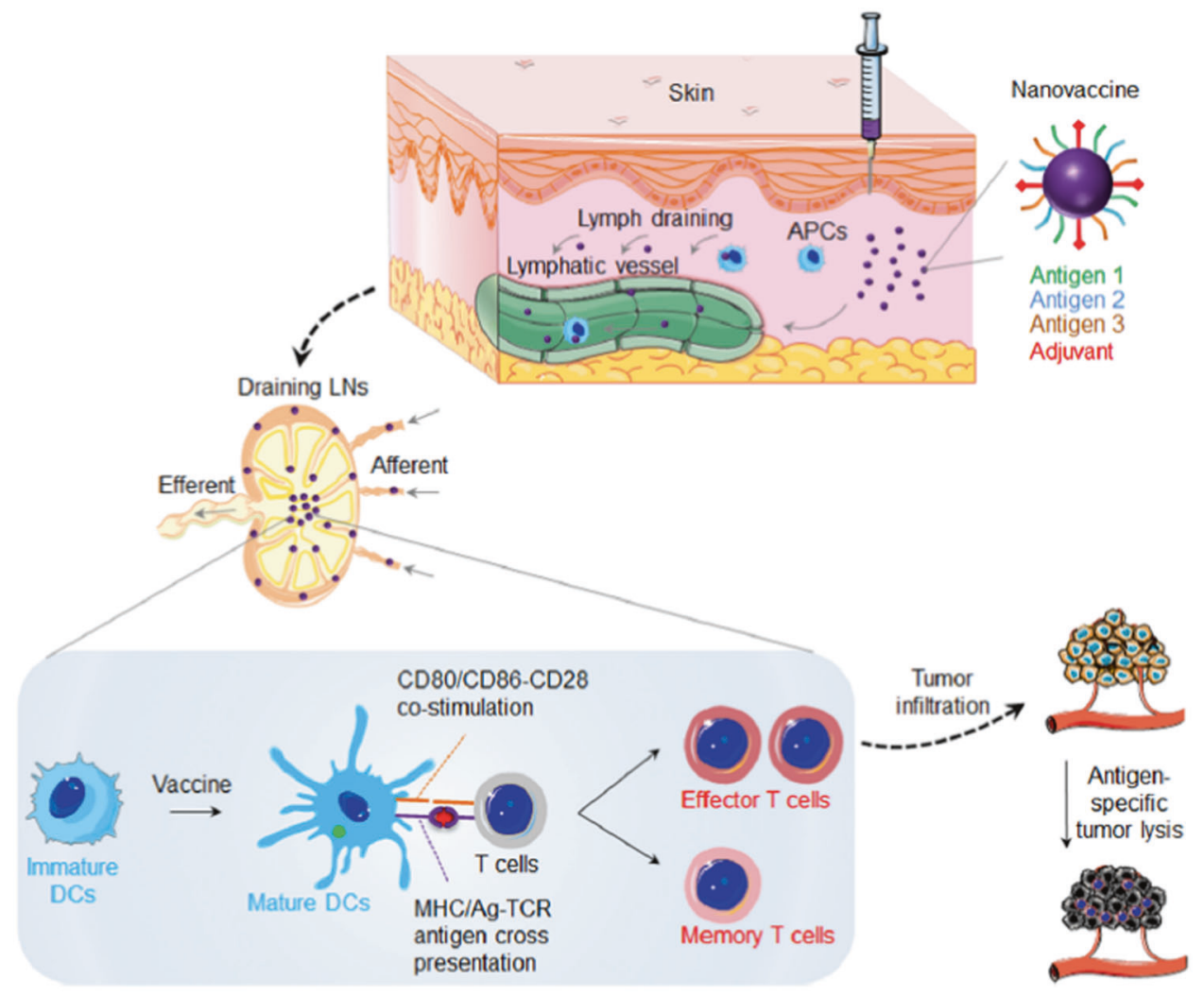

Fig. 1 Schematic of nanovaccine for cancer immunotherapy. Nanovaccines can be loaded with both adjuvant and antigens on the surface (as depicted) or inside nanocarrier. Locally administered nanovaccines efficiently codeliver adjuvant and antigens to lymphoid organs for antigen presentation and induction of robust antitumor T-cell responses. Reprinted with permission from ref. [19] (Copyright 2017, American Chemistry Society).

cell (DC) treatment for glioblastoma (NCT01808820), peptide vaccines for recurrent glioblastoma (NCT02754362) and whole-cell vaccines for breast cancer (NCT00317603), yet the limited ability to generate strong antitumor responses has hindered their wide application [15]. Therapeutic cancer vaccines should induce targeted killing of tumor cells as well as long-lasting immune protection against tumor recurrence or metastasis. High levels of tumor-infiltrating $T$ cells are associated with improved prognosis in many cancers, so it is expected that new-generation vaccines will induce effective Th1 and $\mathrm{CD} 8^{+} \mathrm{CTL}$ responses (Fig. 1) [3, 16-19]. Efficient delivery of antigens and adjuvants to antigenpresenting cells (APCs) in lymphoid organs (peripheral lymph nodes), cytosolic delivery and cross-presentation (complexation with MHC I) of tumor-associated antigens (TAAs), and activation of DCs by appropriate stimulators are desired.

Lymph node-targeting effect

The physical size of the vaccine formulation was found to play an important role in lymph node delivery $[14,20]$. Molecules and particles injected in local tissues are cleared through the blood system if they are smaller than $5 \mathrm{~nm}$. However, particles with hydrodynamic diameters of 10-100 nm can improve the efficiency of lymphatic uptake [21-23]. Reddy et al. exploited poly-(ethylene glycol)-stabilized poly-(propylene sulfide) (PPS) NPs to target lymph nodes, and the results demonstrated that $20 \mathrm{~nm}$ particles drained to the lymphatic system following interstitial injection; NPs between 20 and $45 \mathrm{~nm}$ in size displayed significant accumulation in lymph nodes, showing strong retention at 24, 72,96 , and $120 \mathrm{~h}$ post-injection [24]. Moreover, particles larger than $170 \mathrm{~nm}$ displayed poor lymphatic uptake and strong retention at the injection site [20]. Therefore, nanovaccine development has focused mainly on NPs smaller than $200 \mathrm{~nm}$.

\section{Antigen/adjuvant codelivery}

Compared to traditional vaccines, nanovaccines with controlled size and codelivery of antigen and adjuvant have shown promising effects. Lipid vesicles have attracted much attention for antigen delivery due to their low toxicity and immunogenicity, facile preparation process and reliable manufacturability at commercial scales [25]. James et al. reported a novel design of lipid drug carriers, interbilayer-crosslinked multilamellar vesicles (ICMVs). The multilamellar vesicles stably encapsulate protein antigens in the core, and the immune stimulatory molecules are rapidly released from the vesicles under extracellular conditions. The results showed that these antigen/adjuvant codelivery vesicles could elicit antigen-specific $T$ cells and antibody responses [26]. Liu et al. developed a vaccine comprising lipidmodified antigenic peptides or CpG-DNA, which exploited albumin to transport lipids in vivo and target lymph nodes. The results showed that this nanovaccine produced a 30 -fold increase in $\mathrm{T}$ cell priming and improved antitumor efficacy while apparently reducing systemic toxicity [27]. Additionally, based on this design, $\mathrm{Ma}$ et al. designed amphiphile CAR-T ligands (amph ligands) that, once injected, trafficked to lymph nodes and modified the surfaces of APCs, thus priming CAR-Ts in the lymph node microenvironment. This design induced massive CAR-T expansion and subsequently enhanced antitumor efficacy in multiple mouse tumor models, with low side effects [28].

Polymer-derived nanovaccines allow effective encapsulation and integrated function and are interesting candidates for vaccination purposes. Luo et al. reported that a minimalist nanovaccine, a simple physical mixture of antigens and synthetic polymeric NPs, PC7A NPs $(20-50 \mathrm{~nm})$, can efficiently deliver any tumor-related antigens to APCs in draining lymph nodes while simultaneously stimulating the type I interferon-STING innate 


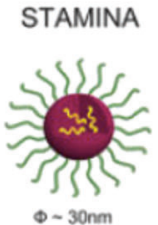

b

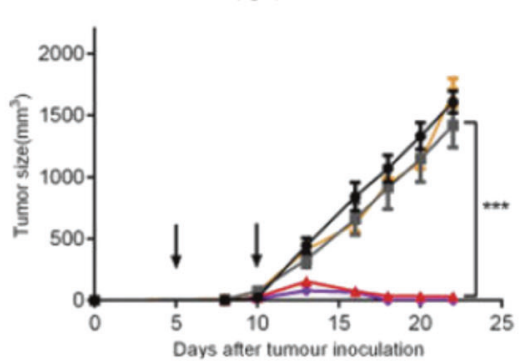

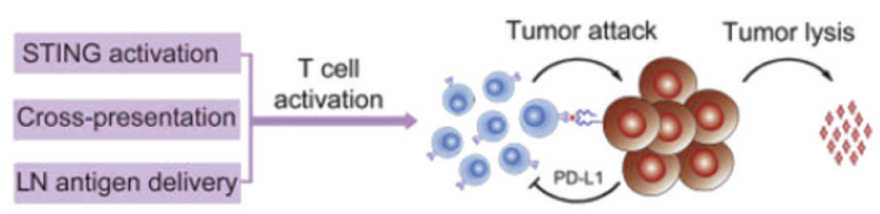

C C TC-1

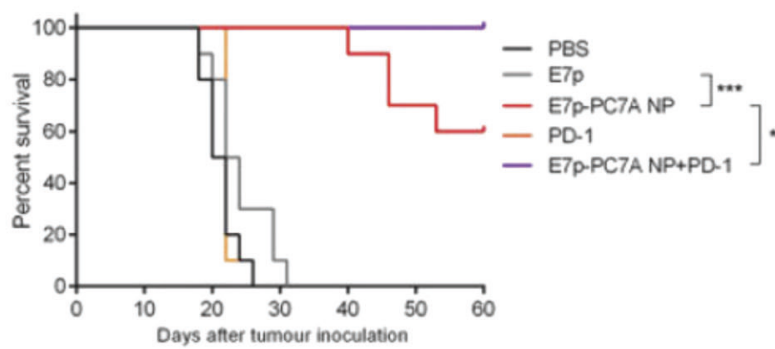

Fig. 2 A STING-activating minimalist nanovaccine (STAMINA) inhibits tumor growth and survival in tumor-bearing mice. a Schematic of STAMINA to boost tumor-specific T cell immunity. In the HPV tumor model, tumor growth inhibition (b) and survival data (c) in C57BL/6 mice showed strong antitumor immunity after tumor inoculation with TC-1 tumor cells. Reprinted with permission from ref. [3] (Copyright 2017, with permission from Elsevier).

immune pathway (Fig. 2). This nanovaccine induced significant immune responses to retard tumor growth in melanoma, colon cancer and human papilloma virus E6/E7 mouse tumor models [29]. Mechanistically, the particle size and simple protein loading strategy facilitate antigen delivery to APCs in lymph nodes, the $\mathrm{pH}$-specific proton sponge effect promotes antigen crosspresentation via membrane disruption, and STING pathway stimulation by this polymer leads to robust APC maturation and subsequent T cell activation. Moreover, Luo et al. demonstrated that nanovaccines combined with radiotherapy could induce a synergistic therapeutic effect in both primary and distal tumors in a STING-dependent manner [30]. Qiao et al. used the flash nanocomplexation (FNC) approach to prepare nanovaccines, and they complexed chitosan and heparin with antigens and adjuvants by electronic affinity. This nanovaccine elicited potent Th1-biased immune responses and showed effective protection against lethal virus challenge [31].

Antigens derived from apoptotic or cancerous cells undergo membrane dynamic curvature and lateral diffusion. This process increases the contact area and multiple interactions of antigens with APCs, which could enhance antigen internalization and crosspresentation [32, 33]. Here, Xia et al. developed a novel Pickering emulsion that is particle stabilized and retains properties of forcedependent deformability and lateral mobility of presented antigens. Hence, this emulsion could improve the recruitment of APCs and enhance antigen uptake and APC activation. The results showed that this vaccine could effectively activate both humoral and cellular responses, further protecting mice from infections and diseases [34].

Biomimetic mimicking delivery

Biomimetic particles represent a promising formulation for the development of safe nanomedicines. Compared to synthetic particles, biomimetic NPs can target tumors or immune cells with prolonged circulating time and low toxicity [35-37].

High-density lipoprotein nanodisks $(\sim 10 \mathrm{~nm})$ are endogenous NPs that transport fats and cholesterol in blood, so they have been clinically tested for drug delivery and show high tolerance [38]. Rui et al. conjugated antigens/adjuvant (Cho-CpG) on these nanodisks, which markedly enhanced the antigen-specific $\mathrm{T}$ cell response. The nanovaccine could eliminate MC-38 and B16F10 tumors when synergized with anti-PD-1 and anti-CTLA-4 therapy [39].
Cell-membrane coating technology is an emerging platform for drug delivery [36]. Cell membrane-coated NPs possess intrinsic properties of source cells, such as a remarkable capacity to contact their surrounding environment due to their biomimetic interface. Compared to traditional nanoparticles, they displayed outstanding characteristics of long circulation and disease-relevant targeting. Since many tumor-related antigens exist on the tumor cell membrane [17, 40], Ashley et al. developed tumor cell membrane-coated NPs containing a highly immunostimulatory adjuvant. The results showed that this nanovaccine could generate a durable anticancer response in vivo and have synergistic effects with checkpoint blockade inhibitors to retard cancer growth [41].

mRNA vaccine

In recent years, messenger RNA (mRNA)-based vaccines have been developed as an alternative prophylactic or therapeutic vaccine technology against infectious diseases or cancers [42]. Compared to traditional DNA vaccines, mRNA vaccines have a safety advantage, as they have very little interaction with the genome. Compared to subunit protein or peptide vaccines, mRNA vaccines are able to deliver multiple antigens in one protein-encoding open reading frame, offering great flexibility and convenience for application and production [43]. Two challenges limit the clinical translation of mRNA vaccines: (1) insufficient cytosolic delivery and (2) rapid degradation with low expression levels. Nanotechnology provides a unique strategy for mRNA delivery $[44,45]$.

Miao et al. designed a library of ionizable lipid-like materials to screen for mRNA delivery vehicles. They identified a lipid vesicle that not only showed high delivery and expression levels but also stimulated the STING innate immune pathway. This formulation could elicit a robust immune response, resulting in inhibition of tumor growth and prolonged survival in melanoma and human papillomavirus E7 tumor models [46].

The cytotoxic $\mathrm{T}$ cell response plays an important role in antitumor immune responses. Oberli et al. developed lipid-based NPs for the delivery of mRNA vaccines to elicit strong cytotoxic $\mathrm{CD}^{+} \mathrm{T}$ cell responses. The vaccine consisted of ionizable lipids, which are positively charged at low $\mathrm{pH}$ and form complexes with negatively charged mRNA. This formulation helps with cellular uptake and cross-presentation of expressed antigens. The results showed that mRNA vaccines led to tumor shrinkage and prolonged the survival of mice (Fig. 3) [47]. 

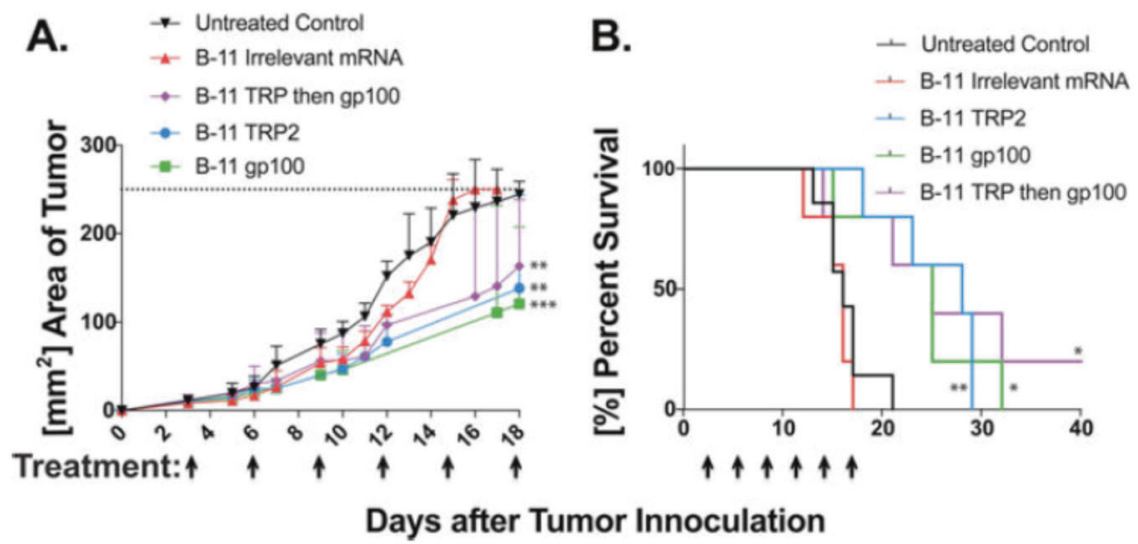

Fig. 3 mRNA LNPs coding for tumor self-antigens, gp100 and TRP2, slow down tumor growth and extend overall survival. a Tumor areas were measured with a caliper lengths $\times$ width. $\mathbf{b}$ All three treated groups survived significantly longer the either the untreated control group or mice treated irrelevant mRNA. Reprinted with permission from ref. [47] (Copyright 2017, American Chemistry Society).

Personalized vaccine design

TAAs are unmutated self-antigens that are highly expressed in tumor tissues but are also expressed in some normal tissues [16]. Two obstacles, central tolerance (low immunogenicity) and aberrant autoimmunity, limit their efficacy and application in cancer therapeutic vaccines $[48,49]$. Recently, the emergence of next-generation sequencing and innovative bioinformatics tools has enabled the discovery of tumor neoantigens. Tumor neoantigens are immunogenic tumor-specific mutations that do not exist in normal tissues $[16,50]$. Personalized vaccines are generated based on neoantigens for the wide variety of tumor mutations in different cancers and persons, which have shown great potential in the clinic.

Patrick et al. incorporated 20 predicted cancer neoantigens present in every person into one formulated vaccine; in a clinical trial, vaccine-induced polyfunctional $\mathrm{CD}^{+}$and $\mathrm{CD}^{+}{ }^{+} \mathrm{T}$ cells targeted $58(60 \%)$ and $15(16 \%)$ of the 97 neoantigens utilized across patients [51].

Glioblastoma (GBM) is the most common and aggressive primary brain tumor [52], which is not sensitive to cancer immunotherapy, especially checkpoint inhibitors. The limited intratumoral infiltration of immune cells and low mutation load (low immunogenicity) lead to a dilemma [53-55]. Norbert et al. reported a clinical program named the Glioma Actively Personalized Vaccine Consortium (GAPVAC) that tested a personalized vaccine in a phase I trial [56]. This vaccine included both unmutated TAAs and neoantigens. The results showed that unmutated APVAC1 antigens activated a sustained central memory $\mathrm{CD}^{+} \mathrm{T}$ cell response and that APVAC2 elicited predominantly $\mathrm{CD}^{+} \mathrm{T}$ helper 1-type $\mathrm{T}$ cell responses against the predicted neoepitopes [56].

Lena et al. reported a vaccine design based on an RNA-lipoplex (RNA-LPX)-based delivery system. By systemically adjusting the net charge of lipid carriers, this vaccine could precisely and efficiently target DCs in vivo via intravenous administration. The results indicated that RNA-LPX-encoded tumor neoantigens or that TAA could elicit potent effector and memory $T$ cell responses as well as retard the progression of tumors in an IFN-alphadependent manner [57].

Min et al. designed a novel antigen-capturing nanoparticle (ACNP) based on PLAG. This NP could capture tumor antigens, especially neoantigens, via surface properties after intratumor injection. It could enhance the uptake of tumor-specific antigens by APCs and promote an antitumor immune response in both injected tumors and distal tumors in melanoma and breast tumor models [58].

In conclusion, nanovaccines are a promising avenue to elicit the $\mathrm{T}$ cell response and inhibit tumor progression. Although great progress has been achieved in nanovaccine design, enormous challenges remain. Facile and stable manufacturing processes, as well as effective clinical translation, are desired.

\section{TUMOR MICROENVIRONMENT-TARGETING NANOMEDICINE}

The tumor microenvironment is a complex cellular environment that is composed of various cells and affects tumor progression [59]. Tumor-associated macrophages (TAMs), myeloid-derived suppressor cells (MDSCs) and T regulatory cells (Tregs) are significant components of the immunosuppressive tumor microenvironment (TME) $[6,60]$. TAMs display an antiinflammatory M2like phenotype to support tumor growth and suppress $\mathrm{CD} 8^{+} \mathrm{T}$ cell recruitment to the tumor microenvironment $[61,62]$. MDSCs play a key role in fighting $\mathrm{T}$ cell activation and the polarization of $\mathrm{M} 1$ macrophages, as well as inhibiting the cytotoxicity of NK cells $[63,64]$. In addition, regulatory T cells (Tregs) are a subset of CD4 ${ }^{+}$ $T$ cells that regulate immune tolerance through various mechanisms [65].

Biophysicochemical differences between tumor and normal tissues, including vascular abnormalities, hypoxia, acidic $\mathrm{pH}$ and alternative metabolic status, also contribute to the immunosuppressive environment in tumors [66,67]. Malignant cells undergo metabolic changes that lead to increased production of lactate, nitric oxide, reactive oxygen species and other byproducts of arachidonic acid metabolism that influence the inflammatory tumor microenvironment [68]. These changes lead to functional programming in TAMs, including the production of cytokines and angiogenetic factors, and result in tumor progression and metastasis. Consumption of the energy sources (e.g., glucose) can support tumor growth, and the glucose metabolism product lactate is an important immunosuppressive metabolite in the tumor microenvironment and a driving factor for TAM-2 polarization [69]. In addition, the hypoxia and low pH of the tumor microenvironment can hinder antitumor immune responses of tumor-infiltrating $\mathrm{T}$ cells by limiting nutrient and energy consumption [70].

Many clinical analyses have indicated that cancer immunotherapy is limited mainly by the immunosuppressive tumor microenvironment [6]. Thus, targeting the tumor microenvironment can help address these obstacles (Fig. 4) [71].

EPR effect

NP drugs can accumulate in tumors for EPR effects, which was first described by Matsumura and Maeda in 1986 [72]. EPR depends on specific pathophysiological traits of tumors. In healthy tissues, small molecules easily leak from the blood vessels, yet NPs cannot due to their size difference. In tumor sites, abnormally broad 


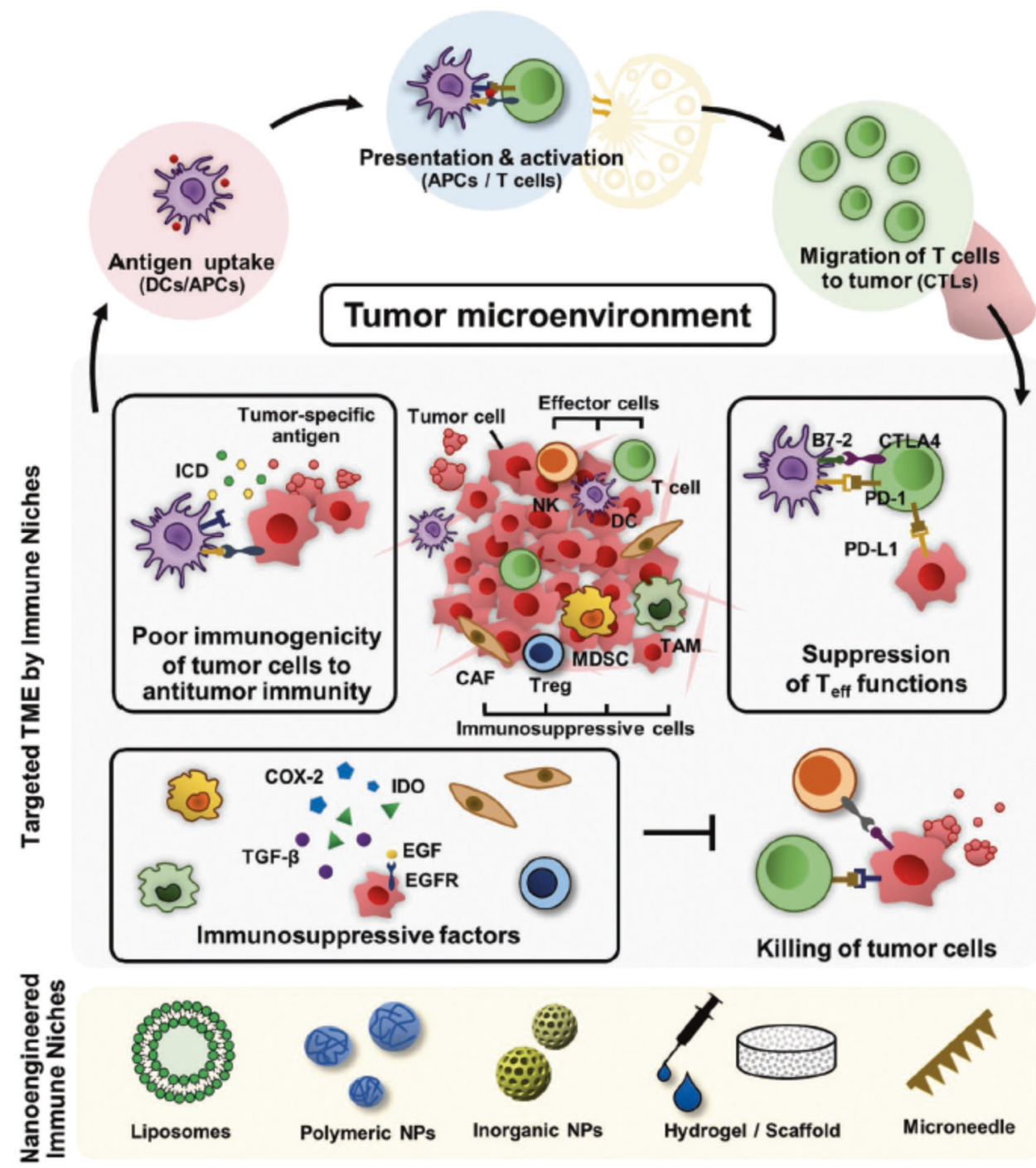

Fig. 4 Schematic of imitation of current cancer immunotherapy, which can be overcome by nanoengineering-based strategies for reprogramming the immunosuppressive TME. Reprinted with permission from ref. [71] (Copyright 2019, John Wiley and Sons).

fenestrations in blood vessels permit extravasation of particles with sizes up to several hundred nanometers [73]. With the absence of lymphatic drainage, NPs show a relatively effective and selective accumulation in tumor tissue, which is 10-200 times higher than that in normal tissues or organs [74]. For example, compared with free doxorubicin, doxorubicin with a liposomal formulation achieves at least a 60 -fold increase in tumors under a concentration-time curve (AUC) [75]. Therefore, nanotechnology shows potential in cancer therapy via EPR-based tumor targeting [76].

Targeting immunosuppressive cells

TAMs displaying an antiinflammatory M2-like phenotype as well as a proinflammatory $\mathrm{M} 1$-like phenotype play a key role in tumor progression, influencing proliferation, metastasis and recurrence [77]. Chen et al. designed an in situ-formed bioresponsive immunotherapeutic nanogel that was preloaded with an antiCD47 antibody in fibrin gel calcium carbonate $\left(\mathrm{CaCO}_{3}\right)$ NPs. The fibrin gel NPs could release anti-CD47 antibodies in acidic tumor surgical wounds, resulting in TAMs polarized to the M1-like proinflammatory phenotype. Activated phagocytes can present antigens and induce a $\mathrm{T}$ cell response against tumor recurrence and metastasis [78].

Wang et al. developed mannose-decorated lactoferrin nanoparticles (Man-LF NPs) and codelivery with the drugs Shikonin and JQ1. Shikonin is isolated from Chinese herbs and shows potential anticancer activity. JQ1 can reduce PD-L1 expression on tumor cells. This formulation managed to target mannose receptorexpressing tumor cells and repolarize TAMs, resulting in remodeling of the TME [79].

Regulatory T cells (Tregs) also contribute to the TME and induce tumor escape [80]. Ou et al. reported thyp 1 peptide-modified hybrid NPs to deliver a drug, IMT, into Tregs in tumors. The peptide thyp 1 could specifically recognize Neuropilin-1 (Nrp1) proteins on Tregs. Combination with anti-CTLA-4 antibodies could modulate the immunosuppressive environment and boost antitumor T cell responses [81].

MDSCs are heterogeneous cells from the myeloid cell lineage that are strongly expanded in cancer and chronic inflammation sites [82]. Yu et al. developed a MDSC membrane-coated iron oxide nanoparticle (MNP@MDSC) to achieve active tumor targeting. The results showed that the formulation could induce 

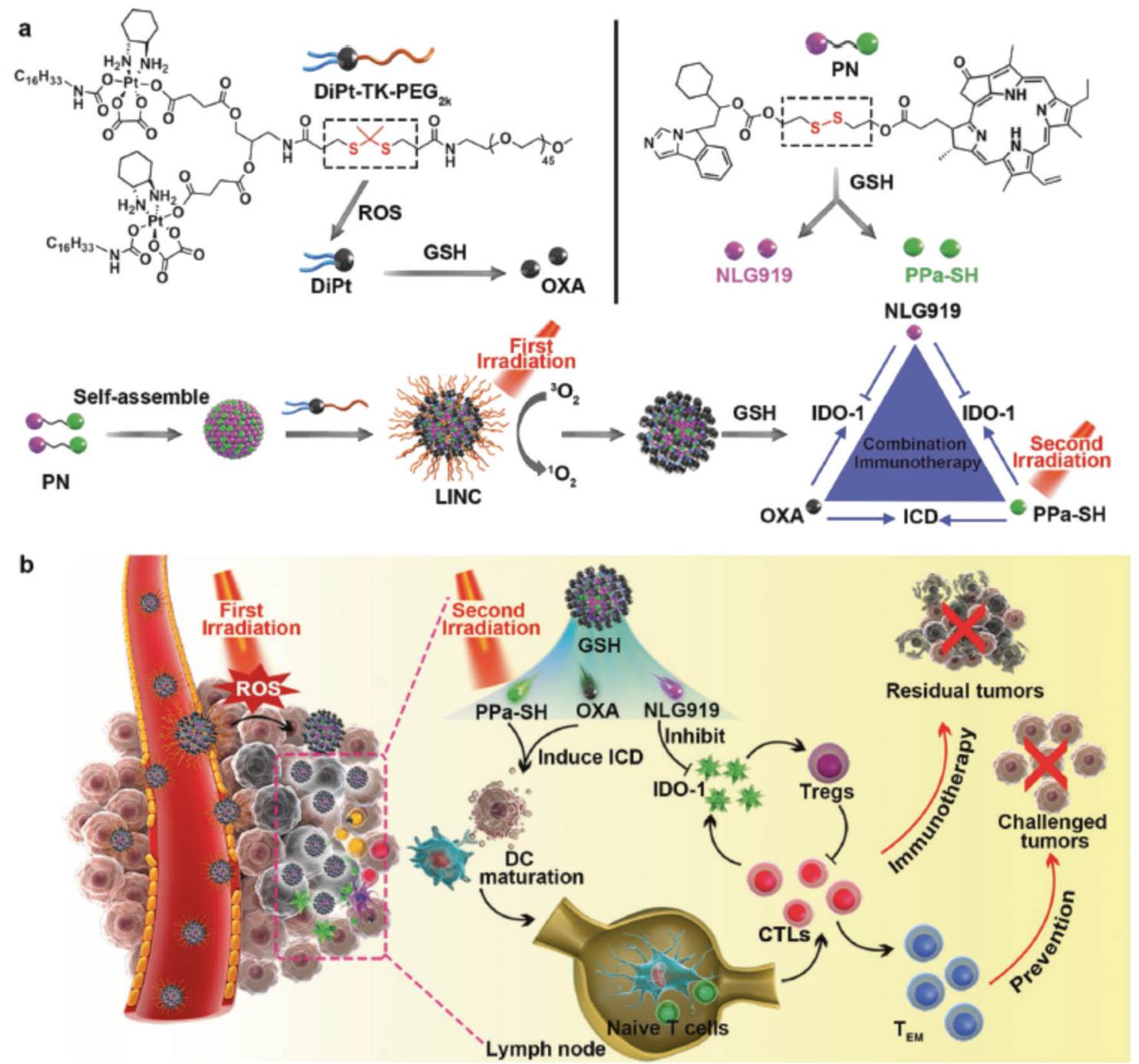

Fig. 5 Schematic illustration of NIR light-inducible LINC (Light-inducible nanocargo) for self-amplified drug delivery and combination immunotherapy. a Fabrication of the light-inducible prodrug nanocargoes LINC. b Schematic illustration of LINC for improved drug delivery and chemoimmunotherapy by eliciting tumor immunogenicity and overcoming immunosuppressive tumor microenvironment. Reprinted with permission from ref. [88] (Copyright 2019, John Wiley and Sons).

PTT-induced immunogenic cell death, reverse macrophage polarization, and reduce tumor metabolic activity.

Targeting the metabolism of the TME

Hypoxia and acidity of the tumor microenvironment are associated with tumor metabolism and can strongly inhibit the antitumor immune response by reducing the cytotoxic activity of CTLs and NK cells $[83,84]$.

Yang et al. reported a $\mathrm{pH}$-responsive nanoplatform that could control the release of the photodynamic agent chlorine e6 (Ce6) and doxorubicin (DOX). This drug formulation could relieve the hypoxic TME by inducing the decomposition of endogenous $\mathrm{H}_{2} \mathrm{O}_{2}$ inside the tumor and remarkably enhance a series of antitumor immune responses.

Tumor cells overexpressing LDHA convert glucose into lactate, display accelerated glycolysis, and cause tumor acidity [85]. Zhang et al. exploited a cationic lipid-assisted NP loaded with LDHA siRNA to knockdown the LDHA gene. By interfering with tumor acidification, this NP decreased the number of immunosuppressive cells, increased the infiltration of $\mathrm{CD}^{+} \mathrm{T}$ cells, and restored antitumor functions [86].
Combination with photothermal therapy to the remodel TME Deep penetration of nanomedicine into tumors remains challenging due to the pathophysiological barrier of solid tumors, so physical intervention may help to overcome this barrier [87]. Feng et al. developed a light-inducible nanocargo (LINC) combined with chemotherapy for immunotherapy. Upon fluorescence activation, the first wave of NIR laser irradiation generated reactive oxygen species (ROS), induced the cleavage of the polyethylene glycol (PEG) coating, and further enhanced tumor retention and deep penetration of the LINC. After exposure to NIR wave laser eradication, the LINC successfully elicited an immune response against tumors (Fig. 5) [88].

Insufficient infiltration of cytotoxic T lymphocytes (CTLs) in the tumor microenvironment is critical for cancer immunological tolerance [89]. Wang et al. reported an engineered MMP-2sensitive nanocomplex loaded with anti-PD-L1 antibodies and the photosensitizer indocyanine green. The NPs could precisely target the tumor site, and near-infrared (NIR) laser irradiation triggered the activation of the photosensitizer and released the anti-PD-L1 antibodies. The results showed that the NPs could increase the 


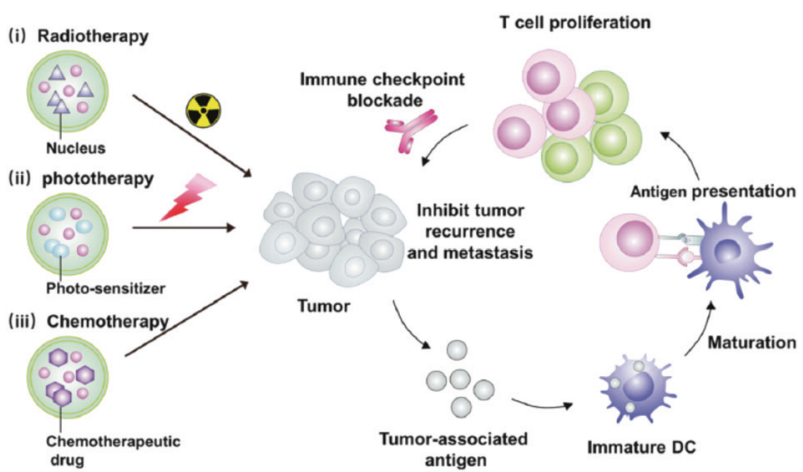

Fig. 6 Local biomaterials-assisted for cancer immunotherapy. (i) radiotherapy activate the immune system, (ii) phototherapy activate the immune system, (iii) chemotherapy activate the immune system, which in combination with immune checkpoint blockade inhibitors could trigger systemic antitumor immunological responses.

frequency of tumor-infiltrating CTLs and promote the effectiveness of PD-L1 blockade therapy [90].

\section{Combination with photodynamic therapy (PDT) to remodel the}

TME

Nanomaterial-based PDT also shows some advantages in antitumor immunotherapy [91]. Meng et al. developed a lighttriggered in situ gelation system composed of photosensitizermodified catalase and poly-(ethylene glycol) double acrylate (PEGDA). Furthermore, adjuvant NPs were introduced into the gelation system. The results showed that local injection of the mixed precursor solution into tumors and subsequent light exposure could trigger robust antitumor immune responses. Accordingly, such a local light-elicited gelation system could effectively trigger antitumor responses via synergistic photoimmunotherapy [92].

Combination with radiation to remodel the TME

Radiotherapy has been broadly applied in clinical cancer treatment; however, the therapeutic effect is limited by tumor hypoxia-associated radiation resistance [91]. Nanomedicine can improve radiotherapy and promote antitumor immune responses. Chen et al. utilized a PLGA-based core-shell nanomaterial to coencapsulate water-soluble catalase (Cat), an enzyme that transforms $\mathrm{H}_{2} \mathrm{O}_{2}$ into $\mathrm{O}_{2}$, and a hydrophobic TLR-7 agonist (R837). The PLGA-R837@Cat NPs could increase radiotherapy efficacy by relieving tumor hypoxia and remodeling the immunosuppressive tumor microenvironment by the Toll-like receptor. Furthermore, the formulation showed a great abscopal effect and long-term immune memory combined with CTLA-4 checkpoint blockade [93].

\section{Combination with chemotherapy}

Chemotherapy is extensively used in the clinic. Some chemotherapeutics (e.g., oxaliplatin, OXA) can induce an antitumor immune response via immunogenic cell death (ICD), but the subsequent immune response is suppressed by immunosuppressive mechanisms (e.g., IDO upregulation for OXA) [94]. Huang et al. reported cationic lipid-assisted nanoparticles (CLANs) loaded with IDO1 siRNA. Coadministration of OXA and $\mathrm{CLAN}_{\text {silDO1 }}$ to tumorbearing mice systemically activated the immune response, improved the frequency of tumor-infiltrating $T$ lymphocytes and decreased the frequency of Tregs in a colorectal tumor model [95].

In summary, the unique characteristics of the tumor microenvironment have been recognized as a considerable target for precise and effective nanomedicine design. Moreover, depending on the regulation of tumor-associated immune cells, the tumor metabolism microenvironment and combination with conventional therapies (especially localized therapeutics), nanomedicine offers a promising platform to remodel the tumor microenvironment, relieving the immunosuppression and evoking antitumor immune responses to destroy cancer cells (Fig. 6) [91].

\section{PERSPECTIVE}

High-efficiency cancer immunotherapy is still challenging due to low response rates, immune evasion or immune resistance. Nanoengineered technology plays a crucial role in overcoming these challenges.

Cancer vaccines have shown feasible therapeutic effects in some preliminary clinical trials. GAPVAC-101, a formulation with lipids, poly $(\mathrm{I}: \mathrm{C})$ and antigens, displayed safety and immunogenicity in a phase I trial [96]. However, further efforts are required to decrease the antigen-screening process. Polymer-based nanomedicines also show potential in cancer immunotherapy, such as PLGA copolymers, which can encapsulate antigens and extend circulation half-life $[97,98]$, as well as improve T cell responses. However, many synthetic or modified materials need be approved through new drug certification; complicated chemistry and multiple components in one formulation may be challenging for clinical translation and CMC (chemistry, manufacturing, and controls). There is a growing demand for researchers to engineer simple nanomedicines and improve the rate of clinical translation.

Small molecules (e.g., inhibitors or agonists), monoclonal antibodies and nanomedicine have shown remarkable potential in cancer immunotherapy [99]. Compared to small molecules, nanomedicine displays potential advantages, such as (1) prolonged circulation half-life [98]; (2) enhanced tumor accumulation; and (3) controlled release and multiple function orchestration by unique design. EPR-based NP delivery also showed potential auxiliary therapeutic effects with surgery, chemotherapy and radiotherapy [98]. However, it has been reported that only $0.7 \%$ (median) of the administered NPs were delivered to solid tumors in patients [100]. Inefficient targeting of NPs in the clinic limits the wide application of nanomedicine. Nanomaterials could also be applied to tumor diagnosis and other types of disease treatment to increase their application field. Additional research will be needed to simplify nanomedicine and improve targeting efficiency, which would lead to more exciting medical breakthroughs.

\section{ADDITIONAL INFORMATION}

Competing interests: The authors declare no competing interests.

\section{REFERENCES}

1. Yousefi H, Yuan J, Keshavarz-Fathi M, Murphy JF, Rezaei N. Immunotherapy of cancers comes of age. Expert Rev Clin Immunol. 2017;13:1001-15.

2. Topalian SL, Drake CG, Pardoll DM. Immune checkpoint blockade: a common denominator approach to cancer therapy. Cancer Cell. 2015;27:450-61.

3. Luo M, Samandi LZ, Wang Z, Chen ZJ, Gao J. Synthetic nanovaccines for immunotherapy. J Control Release. 2017;263:200-10.

4. Wu X, Gu Z, Chen Y, Chen B, Chen W, Weng L, et al. Application of PD-1 blockade in cancer immunotherapy. Comput Struct Biotechnol J. 2019;17:661-74.

5. Sharma P, Hu-Lieskovan S, Wargo JA, Ribas A. Primary, adaptive, and acquired resistance to cancer immunotherapy. Cell. 2017;168:707-23.

6. O'Donnell JS, Teng MWL, Smyth MJ. Cancer immunoediting and resistance to T cell-based immunotherapy. Nat Rev Clin Oncol. 2019;16:151-67.

7. Smyth MJ, Ngiow SF, Ribas A, Teng MW. Combination cancer immunotherapies tailored to the tumour microenvironment. Nat Rev Clin Oncol. 2016;13:143-58.

8. Sultana S, Khan MR, Kumar M, Kumar S, Ali M. Nanoparticles-mediated drug delivery approaches for cancer targeting: a review. J Drug Target. 2013;21:107-25.

9. Grimaldi N, Andrade F, Segovia N, Ferrer-Tasies L, Sala S, Veciana J, et al. Lipidbased nanovesicles for nanomedicine. Chem Soc Rev. 2016;45:6520-45.

10. Obici L, Berk JL, Gonzalez-Duarte A, Coelho T, Gillmore J, Schmidt HH, et al. Quality of life outcomes in apollo, the phase 3 trial of the rnai therapeutic 
patisiran in patients with hereditary transthyretin-mediated amyloidosis. Amyloid. 2020:1-10. https://doi.org/10.1080/13506129.2020.1730790.

11. Zhang Y, Li N, Suh H, Irvine DJ. Nanoparticle anchoring targets immune agonists to tumors enabling anti-cancer immunity without systemic toxicity. Nat Commun. 2018;9:6.

12. van der Meel R, Sulheim E, Shi Y, Kiessling F, Mulder WJM, Lammers T. Smart cancer nanomedicine. Nat Nanotechnol. 2019;14:1007-17.

13. Wagner V, Dullaart A, Bock AK, Zweck A. The emerging nanomedicine landscape. Nat Biotechnol. 2006;24:1211-7.

14. Irvine DJ, Swartz MA, Szeto GL. Engineering synthetic vaccines using cues from natural immunity. Nat Mater. 2013;12:978-90.

15. Kroll AV, Jiang Y, Zhou J, Holay M, Fang RH, Zhang L. Biomimetic nanoparticle vaccines for cancer therapy. Adv Biosyst. 2019;3:1800219. https://doi.org/ 10.1002/adbi.201800219.

16. Hu Z, Ott PA, Wu CJ. Towards personalized, tumour-specific, therapeutic vaccines for cancer. Nat Rev Immunol. 2018;18:168-82.

17. Kroll AV, Fang RH, Zhang L. Biointerfacing and applications of cell membranecoated nanoparticles. Bioconjug Chem. 2017;28:23-32.

18. Kakwere $\mathrm{H}$, Ingham ES, Allen $\mathrm{R}$, Mahakian LM, Tam SM, Zhang $\mathrm{H}$, et al. Toward personalized peptide-based cancer nanovaccines: a facile and versatile synthetic approach. Bioconjug Chem. 2017;28:2756-71.

19. Zhu G, Zhang F, Ni Q, Niu G, Chen X. Efficient nanovaccine delivery in cancer immunotherapy. ACS Nano. 2017;11:2387-92.

20. Bachmann MF, Jennings GT. Vaccine delivery: a matter of size, geometry, kinetics and molecular patterns. Nat Rev Immunol. 2010;10:787-96.

21. Reddy ST, Berk DA, Jain RK, Swartz MA. A sensitive in vivo model for quantifying interstitial convective transport of injected macromolecules and nanoparticles. J Appl Physiol. 2006;101:1162-9.

22. Rohner NA, Thomas SN. Flexible macromolecule versus rigid particle retention in the injected skin and accumulation in draining lymph nodes are differentially influenced by hydrodynamic size. ACS Biomater Sci Eng. 2017;3:153-9.

23. Kaminskas LM, Kota J, McLeod VM, Kelly BD, Karellas P, Porter CJ. Pegylation of polylysine dendrimers improves absorption and lymphatic targeting following SC administration in rats. J Control Release. 2009;140:108-16.

24. Reddy ST, Rehor A, Schmoekel HG, Hubbell JA, Swartz MA. In vivo targeting of dendritic cells in lymph nodes with poly(propylene sulfide) nanoparticles. J Control Release. 2006;112:26-34.

25. Torchilin VP. Recent advances with liposomes as pharmaceutical carriers. Nat Rev Drug Discov. 2005;4:145-60.

26. Moon JJ, Suh H, Bershteyn A, Stephan MT, Liu H, Huang B, et al. Interbilayercrosslinked multilamellar vesicles as synthetic vaccines for potent humoral and cellular immune responses. Nat Mater. 2011;10:243-51.

27. Liu H, Moynihan KD, Zheng Y, Szeto GL, Li AV, Huang B, et al. Structure-based programming of lymph-node targeting in molecular vaccines. Nature. 2014;507:519-22.

28. Ma L, Dichwalkar T, Chang JYH, Cossette B, Garafola D, Zhang AQ, et al. Enhanced car-T cell activity against solid tumors by vaccine boosting through the chimeric receptor. Science. 2019;365:162-8.

29. Luo M, Wang H, Wang Z, Cai H, Lu Z, Li Y, et al. A STING-activating nanovaccine for cancer immunotherapy. Nat Nanotechnol. 2017;12:648-54.

30. Luo M, Liu Z, Zhang X, Han C, Samandi LZ, Dong C, et al. Synergistic STING activation by $P C 7 A$ nanovaccine and ionizing radiation improves cancer immunotherapy. J Control Release. 2019;300:154-60.

31. Qiao D, Liu L, Chen Y, Xue C, Gao Q, Mao HQ, et al. Potency of a scalable nanoparticulate subunit vaccine. Nano Lett. 2018;18:3007-16.

32. Albert ML, Sauter B, Bhardwaj N. Dendritic cells acquire antigen from apoptotic cells and induce class I-restricted ctls. Nature. 1998;392:86-9.

33. Ben M'Barek K, Molino D, Quignard S, Plamont MA, Chen Y, Chavrier $P$, et al. Phagocytosis of immunoglobulin-coated emulsion droplets. Biomaterials. 2015;51:270-7.

34. Xia Y, Wu J, Wei W, Du Y, Wan T, Ma X, et al. Exploiting the pliability and lateral mobility of pickering emulsion for enhanced vaccination. Nat Mater. 2018;17:187-94.

35. Zhu C, Xia Y. Biomimetics: reconstitution of low-density lipoprotein for targeted drug delivery and related theranostic applications. Chem Soc Rev. 2017;46:7668-82.

36. Fang RH, Kroll AV, Gao W, Zhang L. Cell membrane coating nanotechnology. Adv Mater. 2018;30:e1706759.

37. Meyer RA, Sunshine JC, Green JJ. Biomimetic particles as therapeutics. Trends Biotechnol. 2015;33:514-24.

38. Kornmueller K, Vidakovic I, Prassl R. Artificial high density lipoprotein nanoparticles in cardiovascular research. Molecules. 2019;24:2829. https://doi.org/ 10.3390/molecules24152829.

39. Kuai R, Ochyl LJ, Bahjat KS, Schwendeman A, Moon JJ. Designer vaccine nanodiscs for personalized cancer immunotherapy. Nat Mater. 2017;16:489-96.
40. Zhou J, Kroll AV, Holay M, Fang RH, Zhang L. Biomimetic nanotechnology toward personalized vaccines. Adv Mater. 2020;32:e1901255.

41. Kroll AV, Fang RH, Jiang Y, Zhou J, Wei $X, Y u C L$, et al. Nanoparticulate delivery of cancer cell membrane elicits multiantigenic antitumor immunity. Adv Mater. 2017;29;10. https://doi.org/10.1002/adma.201703969.

42. Maruggi G, Zhang C, Li J, Ulmer JB, Yu D. mRNA as a transformative technology for vaccine development to control infectious diseases. Mol Ther. 2019:27:757-72.

43. Pollard C, Rejman J, De Haes W, Verrier B, Van Gulck E, Naessens T, et al. Type I ifn counteracts the induction of antigen-specific immune responses by lipidbased delivery of mRNA vaccines. Mol Ther. 2013;21:251-9.

44. Jayaraman M, Ansell SM, Mui BL, Tam YK, Chen J, Du X, et al. Maximizing the potency of sirna lipid nanoparticles for hepatic gene silencing in vivo. Angew Chem Int Ed Engl. 2012;51:8529-33.

45. Kauffman KJ, Webber MJ, Anderson DG. Materials for non-viral intracellular delivery of messenger RNA therapeutics. J Control Release. 2016;240:227-34.

46. Miao L, Li L, Huang Y, Delcassian D, Chahal J, Han J, et al. Delivery of mRNA vaccines with heterocyclic lipids increases anti-tumor efficacy by STINGmediated immune cell activation. Nat Biotechnol. 2019;37:1174-85.

47. Oberli MA, Reichmuth AM, Dorkin JR, Mitchell MJ, Fenton OS, Jaklenec A, et al. Lipid nanoparticle assisted mRNA delivery for potent cancer immunotherapy. Nano Lett. 2017:17:1326-35.

48. Peng $M$, Mo $Y$, Wang $Y$, Wu $P$, Zhang $Y$, Xiong F, et al. Neoantigen vaccine: an emerging tumor immunotherapy. Mol Cancer. 2019;18:128.

49. Dudley ME, Wunderlich JR, Robbins PF, Yang JC, Hwu P, Schwartzentruber DJ, et al. Cancer regression and autoimmunity in patients after clonal repopulation with antitumor lymphocytes. Science. 2002;298:850-4.

50. Schumacher TN, Schreiber RD. Neoantigens in cancer immunotherapy. Science. 2015;348:69-74.

51. Ott PA, Hu Z, Keskin DB, Shukla SA, Sun J, Bozym DJ, et al. An immunogenic personal neoantigen vaccine for patients with melanoma. Nature. 2017;547:217-21.

52. Zhou W, Wahl DR. Metabolic abnormalities in glioblastoma and metabolic strategies to overcome treatment resistance. Cancers. 2019;11:1231. https://doi. org/10.3390/cancers11091231.

53. Robert C, Long GV, Brady B, Dutriaux C, Maio M, Mortier L, et al. Nivolumab in previously untreated melanoma without braf mutation. $N$ Engl J Med. 2015;372:320-30.

54. Quail DF, Joyce JA. The microenvironmental landscape of brain tumors. Cancer Cell. 2017:31:326-41.

55. Alexandrov LB, Nik-Zainal S, Wedge DC, Aparicio SA, Behjati S, Biankin AV, et al. Signatures of mutational processes in human cancer. Nature. 2013;500:415-21.

56. Hilf N, Kuttruff-Coqui S, Frenzel K, Bukur V, Stevanovifá S, Gouttefangeas C, et al. Actively personalized vaccination trial for newly diagnosed glioblastoma. Nature. 2019;565:240-5.

57. Kranz LM, Diken M, Haas H, Kreiter S, Loquai C, Reuter KC, et al. Systemic RNA delivery to dendritic cells exploits antiviral defence for cancer immunotherapy. Nature. 2016;534:396-401.

58. Min Y, Roche KC, Tian S, Eblan MJ, McKinnon KP, Caster JM, et al. Antigencapturing nanoparticles improve the abscopal effect and cancer immunotherapy. Nat Nanotechnol. 2017;12:877-82.

59. Leffers N, Gooden MJ, de Jong RA, Hoogeboom BN, ten Hoor KA, Hollema $\mathrm{H}_{\text {, }}$ et al. Prognostic significance of tumor-infiltrating T-lymphocytes in primary and metastatic lesions of advanced stage ovarian cancer. Cancer Immunol Immunother. 2009;58:449-59.

60. Groth C, Hu X, Weber R, Fleming V, Altevogt P, Utikal J, et al. Immunosuppression mediated by myeloid-derived suppressor cells (MDSCs) during tumour progression. Br J Cancer. 2019;120:16-25.

61. Petty AJ, Li A, Wang X, Dai R, Heyman B, Hsu D, et al. Hedgehog signaling promotes tumor-associated macrophage polarization to suppress intratumoral cd8+ T cell recruitment. J Clin Invest. 2019;129:5151-62.

62. Yin $W$, Yu X, Kang $X$, Zhao $Y$, Zhao $P$, Jin $H$, et al. Remodeling tumor-associated macrophages and neovascularization overcomes EGFR(t790m) -associated drug resistance by PD-L1 nanobody-mediated codelivery. Small. 2018;14:e1802372.

63. Solito $S$, Bronte V, Mandruzzato $S$. Antigen specificity of immune suppression by myeloid-derived suppressor cells. J Leukoc Biol. 2011;90:31-6.

64. Sinha $\mathrm{P}$, Clements VK, Ostrand-Rosenberg $\mathrm{S}$. Reduction of myeloid-derived suppressor cells and induction of $\mathrm{m} 1$ macrophages facilitate the rejection of established metastatic disease. J Immunol. 2005;174:636-45.

65. Zhang Z, Zhou X. Foxp3 instability helps ttregs distinguish self and non-self Front Immunol. 2019;10:2226.

66. Koyama $\mathrm{S}$, Matsunaga $\mathrm{S}$, Imanishi $\mathrm{M}$, Maekawa $\mathrm{Y}$, Kitano $\mathrm{H}$, Takeuchi $\mathrm{H}$, et al. Tumour blood vessel normalisation by prolyl hydroxylase inhibitor 
repaired sensitivity to chemotherapy in a tumour mouse model. Sci Rep. 2017;7:45621.

67. Dai $Y, X u C$, Sun $X$, Chen $X$. Nanoparticle design strategies for enhanced anticancer therapy by exploiting the tumour microenvironment. Chem Soc Rev. 2017;46:3830-52.

68. Netea-Maier RT, Smit JWA, Netea MG. Metabolic changes in tumor cells and tumor-associated macrophages: a mutual relationship. Cancer Lett. 2018;413:102-9.

69. Turbitt WJ, Demark-Wahnefried W, Peterson CM, Norian LA. Targeting glucose metabolism to enhance immunotherapy: emerging evidence on intermittent fasting and calorie restriction mimetics. Front Immunol. 2019;10:1402.

70. Jing X, Yang F, Shao C, Wei K, Xie M, Shen H, et al. Role of hypoxia in cancer therapy by regulating the tumor microenvironment. Mol Cancer. 2019;18:157.

71. Phuengkham H, Ren L, Shin IW, Lim YT. Nanoengineered immune niches for reprogramming the immunosuppressive tumor microenvironment and enhancing cancer immunotherapy. Adv Mater. 2019;31:e1803322.

72. Matsumura $\mathrm{Y}$, Maeda H. A new concept for macromolecular therapeutics in cancer chemotherapy: mechanism of tumoritropic accumulation of proteins and the antitumor agent smancs. Cancer Res. 1986;46:6387-92.

73. Golombek SK, May JN, Theek B, Appold L, Drude N, Kiessling F, et al. Tumor targeting via EPR: strategies to enhance patient responses. Adv Drug Deliv Rev. 2018;130:17-38.

74. Torchilin V. Tumor delivery of macromolecular drugs based on the EPR effect. Adv Drug Deliv Rev. 2011;63:131-5.

75. Gabizon A, Shmeeda H, Barenholz Y. Pharmacokinetics of pegylated liposomal doxorubicin: review of animal and human studies. Clin Pharmacokinet. 2003;42:419-36

76. Kang H, Rho S, Stiles WR, Hu S, Baek Y, Hwang DW, et al. Size-dependent EPR effect of polymeric nanoparticles on tumor targeting. Adv Health Mater. 2020;9: e1901223.

77. Ovais $M$, Guo M, Chen C. Tailoring nanomaterials for targeting tumor-associated macrophages. Adv Mater. 2019;31:e1808303.

78. Chen $Q$, Wang $C$, Zhang $X$, Chen $G, H u$, Li H, et al. In situ sprayed bioresponsive immunotherapeutic gel for post-surgical cancer treatment. Nat Nanotechnol. 2019;14:89-97.

79. Wang $H$, Tang $Y$, Fang $Y$, Zhang $M$, Wang $H$, He Z, et al. Reprogramming tumor immune microenvironment (TIME) and metabolism via biomimetic targeting codelivery of Shikonin/JQ1. Nano Lett. 2019;19:2935-44.

80. Facciabene A, Motz GT, Coukos G. T-regulatory cells: key players in tumor immune escape and angiogenesis. Cancer Res. 2012;72:2162-71.

81. Ou W, Thapa RK, Jiang L, Soe ZC, Gautam M, Chang JH, et al. Regulatory T celltargeted hybrid nanoparticles combined with immuno-checkpoint blockage for cancer immunotherapy. J Control Release. 2018;281:84-96.

82. Ostrand-Rosenberg S, Sinha P, Beury DW, Clements VK. Cross-talk between myeloid-derived suppressor cells (MDSC), macrophages, and dendritic cells enhances tumor-induced immune suppression. Semin Cancer Biol. 2012;22:275-81.

83. Barsoum IB, Smallwood CA, Siemens DR, Graham CH. A mechanism of hypoxiamediated escape from adaptive immunity in cancer cells. Cancer Res. 2014;74:665-74.
84. Huber V, Camisaschi C, Berzi A, Ferro S, Lugini L, Triulzi T, et al. Cancer acidity: an ultimate frontier of tumor immune escape and a novel target of immunomodulation. Semin Cancer Biol. 2017;43:74-89.

85. Yang $G, X u L$, Chao $Y, X u$ J, Sun $X, W u ~ Y$, et al. Hollow $M n O(2)$ as a tumormicroenvironment-responsive biodegradable nano-platform for combination therapy favoring antitumor immune responses. Nat Commun. 2017:8:902.

86. Zhang YX, Zhao YY, Shen J, Sun X, Liu Y, Liu H, et al. Nanoenabled modulation of acidic tumor microenvironment reverses anergy of infiltrating $T$ cells and potentiates anti-PD-1 therapy. Nano Lett. 2019;19:2774-83.

87. MacEwan SR, Chilkoti A. From composition to cure: a systems engineering approach to anticancer drug carriers. Angew Chem Int Ed Engl. 2017;56:6712-33

88. Feng B, Hou B, Xu Z, Saeed M, Yu H, Li Y. Self-amplified drug delivery with lightinducible nanocargoes to enhance cancer immunotherapy. Adv Mater. 2019;31: e1902960.

89. Zhao X, Subramanian S. Intrinsic resistance of solid tumors to immune checkpoint blockade therapy. Cancer Res. 2017;77:817-22.

90. Wang D, Wang T, Yu H, Feng B, Zhou L, Zhou F, et al. Engineering nanoparticles to locally activate T cells in the tumor microenvironment. Sci Immunol. 2019;4: eaau6584. https://doi.org/10.1126/sciimmunol.aau6584.

91. Chen Q, Chen M, Liu Z. Local biomaterials-assisted cancer immunotherapy to trigger systemic antitumor responses. Chem Soc Rev. 2019;48:5506-26.

92. Meng Z, Zhou X, Xu J, Han X, Dong Z, Wang H, et al. Light-triggered in situ gelation to enable robust photodynamic-immunotherapy by repeated stimulations. Adv Mater. 2019;31:e1900927.

93. Chen $\mathrm{Q}$, Chen J, Yang Z, Xu J, Xu L, Liang $C$, et al. Nanoparticle-enhanced radiotherapy to trigger robust cancer immunotherapy. Adv Mater. 2019;31: e1802228.

94. Zhou F, Feng B, Yu H, Wang D, Wang T, Ma Y, et al. Tumor microenvironmentactivatable prodrug vesicles for nanoenabled cancer chemoimmunotherapy combining immunogenic cell death induction and cd47 blockade. Adv Mater. 2019;31:e1805888.

95. Huang H, Jiang CT, Shen S, Liu A, Gan YJ, Tong QS, et al. Nanoenabled reversal of ido1-mediated immunosuppression synergizes with immunogenic chemotherapy for improved cancer therapy. Nano Lett. 2019;19:5356-65.

96. Hilf N, Kuttruff-Coqui S, Frenzel K, Bukur V, Stevanovifá S, Gouttefangeas C, et al. Publisher correction: Actively personalized vaccination trial for newly diagnosed glioblastoma. Nature. 2019;566:E13.

97. Sah H, Thoma LA, Desu HR, Sah E, Wood GC. Concepts and practices used to develop functional plga-based nanoparticulate systems. Int J Nanomed. 2013;8:747-65.

98. Bazile D, Prud'homme C, Bassoullet MT, Marlard M, Spenlehauer G, Veillard M. Stealth me. PEG-PLA nanoparticles avoid uptake by the mononuclear phagocytes system. J Pharm Sci. 1995;84:493-8.

99. Bianchini G, Gianni L. The immune system and response to HER2-targeted treatment in breast cancer. Lancet Oncol. 2014;15:e58-68.

100. Muhamad N, Plengsuriyakarn T, Na-Bangchang K. Application of active targeting nanoparticle delivery system for chemotherapeutic drugs and traditional/herbal medicines in cancer therapy: a systematic review. Int J Nanomed. 2018;13:3921-35 